\section{Mehr Asthma durch Arbeitsstress?}

\section{Schichtarbeit, Termindruck, Konkurrenz: einer von vier Berufstätigen in Deutschland gibt an, permanent unter Stress zu stehen. Dadurch steigt das Risiko für kardiovaskuläre und psychische Erkrankungen. Gibt es auch einen Zusammenhang mit Asthma bronchiale?}

D ie Pathogenese des Asthma bronchiale ist vielschichtig, auch psychosoziale Faktoren könnten eine Rolle spielen. Arbeitsmediziner aus Heidelberg versuchten jetzt erstmals einem Zusammenhang zwischen der Arbeitsbelastung und der Prävalenz sowie Inzidenz von Asthma bronchiale bei Erwachsenen auf die Spur zu kommen. Dazu dokumentierten sie über eine Zeitspanne von durchschnittlich 8,5 Jahren das Ausmaß der beruflichen Belastung und das Auftreten von Asthma bei 5.114 Männern und Frauen im Alter zwischen 40 und 65 Jahren.

$\mathrm{Zu}$ Untersuchungsbeginn hatten 5,6\% der Teilnehmer angegeben, unter Asthma zu leiden. Ein überdurchschnittlich starker Arbeitsstress und das Unvermögen nach der Arbeit abzuschalten und die Freizeit zu genießen, waren

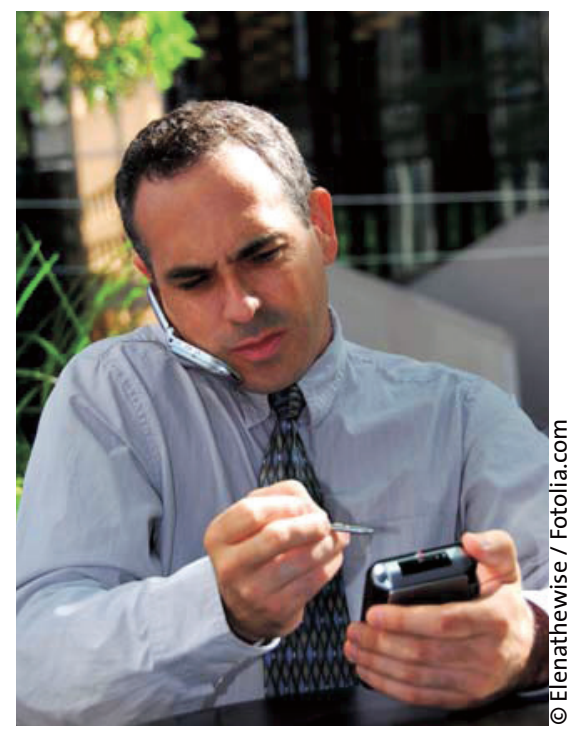

Möglicherweise nicht nur ein Kandidat für ein koronares Ereignis, sondern auch für eine Asthmaexazerbation.

\title{
Kunsttherapie hilft Kindern mit Asthma
}

\section{Patienten mit chronischen Erkrankungen profitieren häufig von einer Kunsttherapie, die sie anleitet, Gefühle und Gedanken etwa durch Malen oder Modellieren auszudrücken. Eine Studie prüfte die Effektivität dieses psychotherapeutischen Ansatzes erstmals bei Kindern mit Asthma.}

ine chronische Atemwegserkran-
kung hinterlässt nicht nur körper-
liche Spuren, auch die psychische Ent-
wicklung asthmakranker Kinder kann
beeinträchtigt sein. Häufiges Fehlen in
der Schule, eingeschränkte Freizeitakti-
vitäten und emotionaler Stress während
der Krankheitsepisoden können die
Ausbildung eines gesunden „Selbstkon-
zeptes“ erschweren und Angst, Depres-
sionen und Verhaltensauffälligkeiten
auslösen.

Im Rahmen einer randomisierten kontrollierten Studie besuchte die
Hälfte der teilnehmenden 22 Kinder zwischen sieben und 14 Jahren mit schwerem, chronischem Asthma einmal wöchentlich für eine Stunde eine kunsttherapeutischen Sitzung. Sieben Wochen lang hatten die Kinder die Möglichkeit, ihre Gefühle und Probleme, die mit ihrer Erkrankung einhergehen, künstlerisch auszudrücken und mit den anderen Betroffen und Therapeuten darüber ins Gespräch zu kommen.

Vor dem Beginn der Kunsttherapie, direkt im Anschluss und nach sechs dabei positiv mit der Asthmaprävalenz assoziiert (Prevalence Ratio [PR] 1,15; 95\%-Konfidenzintervall [KI] 0,97-1,36 bzw. PR 1,43; 95\%-KI 1,12-1,83).

Innerhalb des Untersuchungszeitraumes erkrankten 1,9 \% der Studienteilnehmer neu an Asthma. Dabei hatten die Berufstätigen mit einer hohen Arbeitsbelastung ein doppelt so hohes Risiko, erstmals einen Asthmaanfall zu erleiden wie die Menschen, die im Berufsalltag unter keinem hohen Druck standen.

Die Frage nach den Mechanismen, die Arbeitsstress und Asthmaentstehung verbinden, müssen die Autoren unbeantwortet lassen, vermuten aber, dass die dauerhafte Ausschüttung von Stresshormonen das Immunsystem schwächt und somit eine Entzündung der Atemwege begünstigt.

Fazit: Menschen, die durch ihre Berufstätigkeit einer hohen Stressbelastung ausgesetzt sind, haben ein erhöhtes Risiko an Asthma zu erkranken.

uge

Loerbroks A et al. Work-related stress, inability to relax after work an risk of adult asthma: a population-based cohort study. Allergy 2010; 65: 1298-305

Monaten wurden mit Hilfe standardisierter Methoden bei allen Kindern Lebensqualität, Gefühlslage und Verhaltensweisen bestimmt. Dabei zeigten die Kinder, die die Therapiestunde besucht hatten, unmittelbar danach im Vergleich zur Kontrollgruppe eine deutliche Steigerung des positiven Selbstwertgefühls: Direkt nach den sieben Wochen Kunsttherapie, aber auch noch sechs Monate später war zudem die Lebensqualität signifikant angestiegen und Angstgefühle waren seltener.

Fazit: Kinder mit chronischem Asthma profitieren von einer Kunsttherapie. Auch noch ein halbes Jahr nach den Sitzungen haben sie weniger Ängste und eine verbesserte Lebensqualität. uge

Beebe $\mathbf{A}$ et al. A randomized trial to test the effectiveness of art therapy for children with asthma. J Allergy Clin Immunol 2010; 126: 263-6 